INPLASY

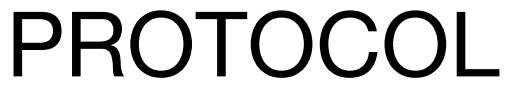

To cite: Chen et al. Association between IL-17A rs2275913 polymorphism and rheumatoid arthritis susceptibility: a metaanalysis. Inplasy protocol 202170056. doi:

10.37766/inplasy2021.7.0056

Received: 18 July 2021

Published: 18 July 2021

Corresponding author: Jing Li

lijing980@yeah.net

Author Affiliation:

Daping Hospital, the Third Affiliated Hospital of Third Military Medical University.

Support: None.

Review Stage at time of this submission: Completed but not published.

Conflicts of interest:

None declared.

\section{Association between IL-17A rs2275913 polymorphism and rheumatoid arthritis susceptibility: a meta-analysis}

Chen, P1; Li, LL2; Zhang, GX3; Zhang, F4; Tang, Y5; Zhou, L6; Yang; $\mathrm{Y}^{7} ; \mathrm{Li}, \mathrm{J}^{8}$.

Review question / Objective: A number of studies have investigated the association of IL-17A rs2275913 polymorphism with susceptibility to rheumatoid arthritis (RA); however, the results remain inconsistent. We conducted this meta-analysis to investigate the relationship between IL-17A rs2275913 polymorphism and RA risk.

Condition being studied: The association between rheumatoid arthritis (RA) susceptibility and IL-17A rs2275913 polymorphism has been revealed by growing evidence from recent studies, but the results remained controversial. Here, we conducted a meta-analysis to summarize and estimate the relationship. A total of eleven publications with 4019 RA patients and 4137 controls were included in the present metaanalysis. The results indicated that IL-17A rs2275913 polymorphism was significantly associated with RA susceptibility in overall population (A vs. G: OR $=0.89,95 \% \mathrm{Cl}$ : 0.83-0.95, $\mathrm{P}<0.001$; GA vs. GG: $\mathrm{OR}=0.87,95 \% \mathrm{Cl}: 0.78-0.96$, $P=0.006$; $A$ A vs. $G G:$ $O R=0.82,95 \% \mathrm{Cl}: 0.71-0.96, P=0.01$; $\mathrm{GA}+\mathrm{AA}$ vs. $\mathrm{GG}: \mathrm{OR}=0.86,95 \% \mathrm{Cl}: 0.78-0.94, \mathrm{P}=0.002)$.

INPLASY registration number: This protocol was registered with the International Platform of Registered Systematic Review and Meta-Analysis Protocols (INPLASY) on 18 July 2021 and was last updated on 18 July 2021 (registration number INPLASY202170056).

\section{INTRODUCTION}

Review question / Objective: A number of studies have investigated the association of IL-17A rs2275913 polymorphism with susceptibility to rheumatoid arthritis (RA); however, the results remain inconsistent. We conducted this meta-analysis to investigate the relationship between IL-17A rs2275913 polymorphism and RA risk.

Rationale: Recent years, the associations between six IL-17A polymorphisms and RA risk are studied by many researchers in different population, and the most popularity variant is IL-17A (rs2275913, 
G-197A). Although some studies showed that IL-17A rs2275913 variant allele reduced the RA risk, the reports that IL-17A rs2275913 variant allele was not important factor to the RA susceptibility are also existed. The results remain inconclusive among the studies. Therefore, we conducted the present meta-analysis to evaluate the association between the IL-17A rs2275913 polymorphism and RA risk.

Condition being studied: The association between rheumatoid arthritis (RA) susceptibility and IL-17A rs2275913 polymorphism has been revealed by growing evidence from recent studies, but the results remained controversial. Here, we conducted a meta-analysis to summarize and estimate the relationship. $A$ total of eleven publications with 4019 RA patients and 4137 controls were included in the present meta-analysis. The results indicated that IL-17A rs2275913 polymorphism was significantly associated with RA susceptibility in overall population (A vs. G: OR $=0.89,95 \% \mathrm{Cl}: 0.83-0.95, \mathrm{P}<$ 0.001 ; GA vs. GG: $O R=0.87,95 \% \mathrm{Cl}$ : $0.78-0.96, P=0.006$; $A A$ vs. $G G: O R=0.82$, 95\% Cl: $0.71-0.96, P=0.01$; GA+AA vs. GG: $\mathrm{OR}=0.86,95 \% \mathrm{Cl}: 0.78-0.94, \mathrm{P}=0.002$ ).

\section{METHODS}

Search strategy: A systematic search in PubMed, Embase, and Web of science online databases was performed by two independent investigators up to June 1 , 2020. The following terms were used: "Interleukin-17A or IL-17A", "genetic polymorphisms or polymorphism or variant" and "rheumatoid arthritis or RA", without any other limitation of origin, languages and items. References of relevant studies, previous meta-analyses and reviews were also manually searched.

Participant or population: A total of $\mathbf{4 0 1 9}$ rheumatoid arthritis patients and 4137 controls were included in this metaanalysis.

Intervention: Rheumatoid arthritis patients.
Comparator: Healthy controls.

Study designs to be included: Case-control studies.

Eligibility criteria: (1) case-control studies; (2) evaluation of the association between IL-17A polymorphisms and RA susceptibility; (3) studies focusing on human being; (4) detailed genotype distribution data could be acquired to calculate odds ratios (ORs) and $95 \%$ confidence intervals(Cls).

Information sources: The data that support the findings of this study are available from the corresponding author upon reasonable request. Jing Li, E-mail: lijing980@yeah.net, Department of Rheumatology, Daping Hospital, the Third Affiliated Hospital of Third Military Medical University, No 10, Daping Changjiang Branch Road, Yuzhong District, Chongqing 400042, China. Phone: 0086-2368757745.

Main outcome(s): The results showed a significant association between IL-17A rs2275913 polymorphism and RA susceptibility in overall population (A vs. G: OR=0.89, 95\% Cl: 0.83-0.95, P<0.001; GA vs. $G G$ : $O R=0.87,95 \%$ Cl: $0.78-0.96$, $P=0.006$; AA vs. GG: $O R=0.82,95 \% \mathrm{Cl}: 0.71-$ $0.96, P=0.01$; $G A+A A$ vs. $G G: O R=0.86,95 \%$ Cl: 0.78-0.94, $P=0.002$ ).

Additional outcome(s): In subgroup analyses, IL-17A rs2275913 polymorphism was significantly associated with RA risk in Europeans (A vs. G: OR=0.87, $95 \% \mathrm{Cl}$ : $0.78-$ $0.97, P=0.01$; $G A$ vs. $G G: O R=0.79,95 \% \mathrm{Cl}$ : $0.68-0.93, P=0.005 ; G A+A A$ vs. $G G$ : OR=0.79, 95\% Cl: 0.68-0.92, $P=0.003$ ), but not in Africans or Americans.

Data management: The name of first author, year of publication, country of origin, the criteria of RA, number of cases and controls, genotype frequency in cases and controls were extracted from eligible study. Two independent authors checked all the collected data and reached consensus on each item. Any encountered discrepancies were resolved by consensus. Data were saved and dealed with Excel. 
Quality assessment / Risk of bias analysis: Eligible studies were critically assessed according to Newcastle-Ottawa Quality AssessmentScale (NOS) for cohort studies with moderate modification. Each included study was judged on 3 main perspectives: (1) the selection of the study groups, (2) the comparability of the groups and (3) the outcome of the groups. Study with a score of 6 or higher was considered as high quality.

Strategy of data synthesis: Chi-square test in control groups was implemented to evaluate the Hardy-Weinberg equilibrium (HWE) of each study, and the HWE was considered a significant departure when $\mathrm{P} 50 \%$ and $\mathrm{P}<0.1$. Random-effects model or a fixed-effect model was used to pool the effect sizes depending on the existence of statistical heterogeneity or not, respectively. Potential publication bias was assessed by Egger's regression test. All statistical tests were performed by the Review Manager software version 5.2 and Stata 12.0 software.

Subgroup analysis: Subgroup analyses based on ethnicity were carried out separately.

Sensitivity analysis: Sensitivity analysis was conducted to examine the influence of the individual article on the pooled effects for IL-17A rs2275913 polymorphism by omitting one study at one time.

Language: No language limitation.

Country(ies) involved: China.

Other relevant information: None.

Keywords: Rheumatoid arthritis; IL-17A; polymorphism; meta-analysis.

Dissemination plans: Publication.

Contributions of each author:

Author 1 - Ping Chen.

Author 2 - Liangliang Li.

Author 3 - Guixin Zhang.

Author 4 - Feng Zhang.

Author 5 - Yan Tang.
Author 6 - Li Zhou.

Author 7 - Yi Yang.

Author 8 - Jing Li. 